\section{Role of factor VIIl-binding capacity of endogenous von Willebrand factor in the development of factor VIII inhibitors in patients with severe hemophilia A}

The development of factor VIII (FVIII) inhibitors is the major complication of replacement therapy in patients with severe hemophilia A. Experimental and clinical evidence suggests that the presence of exogenous von Willebrand factor (VWF) in FVIII products reduces the immunogenicity of therapeutic FVIII. ${ }^{1-3}$ However, a direct immuno-protective effect of endogenous VWF remains unclear. ${ }^{4,5}$ The binding of VWF to FVIII involves the first 272 amino acids of the mature VWF (D'-D3 region) encoded by exons 18-23 of the VWF gene. ${ }^{6}$ Mutations in the VWF gene that result in quantitative or qualitative defects in VWF lead to von Willebrand disease (VWD). Polymorphisms in the VWF gene have been studied in the context of venous thrombosis and VWD, ${ }^{7,8}$ but, to our knowledge, not in that of hemophilia A. Here, we investigated whether the capacity of endogenous VWF in patients with severe hemophilia A to modulate inhibitor development depends on its capacity to bind to therapeutic FVIII. Our working hypothesis was that gene variations in the D'-D3 region result in qualitative changes in the capacity of circulating endogenous VWF to bind FVIII. While such polymorphisms do not translate into coagulation abnormalities, they might have an impact on the stabilization of the therapeutically administered exogenous FVIII in patients with hemophilia A. The consequence would be an increased ratio of free versus bound FVIII molecules and a potentially reduced immuno-protection of FVIII by VWF. Our results show that the relative binding of endogenous VWF to therapeutic FVIII is a poor predictor of inhibitor development, probably reflecting the multi-causal nature of the inhibitor risk. ${ }^{9,10}$

We first evaluated the capacity of endogenous VWF in the plasma of 48 randomly selected patients with severe hemophilia A to bind recombinant FVIII in vitro. VWF:Ag levels were $89.8 \%$ [standard error of mean (SEM): 10.4] and $91.9 \%$ (SEM: 13.0) for inhibitor-positive and inhibitor-negative patients, respectively [95\% confidence interval $(95 \% \mathrm{CI}):-30.9$ to 35.0$]$. The relative VWF binding to FVIII (referred to as VWF:FVIIIB) was determined in each sample using an immuno-assay initially validated for the diagnosis of type 2N VWD (see the Online Supplementary Methods). VWF:FVIIIB was normally distributed and ranged between $41.1 \%$ and $158.9 \%$. Interestingly, the distribution of VWF:FVIIIB was different for inhibitor-positive and negative patients (Figure 1A) with means of $86.4 \%$ (SEM: 5.1) for inhibitor-positive patients as opposed to $103.6 \%$ (SEM: 5.8) for inhibitor-negative patients (95\% CI 1.3-33.2). The receiver operating characteristic curve of VWF:FVIIIB as a predictor of inhibitor development in patients with severe hemophilia A yielded an area under the curve of 0.668 (95\% CI 0.513-0.821) (Figure 1B). Upon examination of the coordinates of the receiver operating characteristic curve, we chose a potential VWF:FVIIIB cutoff value of $95 \%$, which yielded the best relation between sensitivity and specificity. Using this cutoff value, a VWF:FVIIIB below 95\% was more frequent among inhibitor-positive patients than among inhibitor-negative patients $(71 \% v s$. $37 \%$ ), and a value below this cutoff was associated with a more than 4-fold increased risk of inhibitor development (odds ratio, 4.3; 95\% CI: 1.3-14.5). The proposed cutoff value had a sensitivity of 0.71 (95\% CI: $0.48-0.89$ ) and specificity of 0.63 (95\% CI: $0.42-0.81$ ). The calculated positive and negative predictive values for the prediction of inhibitor development were 0.4 and 0.83 , respectively, using an inhibitor prevalence of $30 \%$. These data suggest the potential of the VWF:FVIIIB assay in the preventive identification of patients with severe hemophilia $A$ at a low risk of developing inhibitors during FVIII replacement therapy. It is noteworthy, however, that a substantial number (38\%) of the inhibitor-negative patients included in the study had VWF:FVIIIB scores lower than the median of the whole population; conversely, $35 \%$ of the inhibitor-positive patients had VWF:FVIIIB scores higher than the median. These results highlight the multi-causal nature of inhibitor development.

Exons 18 to 23 were directly sequenced in order to characterize single nucleotide polymorphisms (SNP) in the VWF gene from the 48 patients previously tested for VWF:FVIIIB (Online Supplementary Methods). Four SNP were identified with prevalences equivalent to those previously described in different non-hemophilic populations: ${ }^{\circ}$ c.2365 A $>$ G, p.Thr789Ala (rs1063856); c.2385

Table 1. Distribution of the c.2555 G>A genotypes and their associations with the development of factor VIII inhibitor in 235 patients with severe hemophilia A from the SIPPET study. ${ }^{2}$

\begin{tabular}{|c|c|c|c|c|c|c|}
\hline \multirow[t]{2}{*}{ rFVIII + pdFVIII } & \multirow[t]{2}{*}{ Inh-negative (n=163) } & \multicolumn{3}{|c|}{ Inh-positive (n=72) } & \multirow[b]{2}{*}{ OR } & \multirow[b]{2}{*}{$95 \%$ Cl } \\
\hline & & $\operatorname{LR}(n=24)$ & HR $(n=48)$ & $L R+H R(n=72)$ & & \\
\hline $\mathrm{G} / \mathrm{G}$ & $129(79 \%)$ & 22 & 40 & $62(86 \%)$ & 0.61 & $0.28-1.32$ \\
\hline $\mathrm{G} / \mathrm{A}+\mathrm{A} / \mathrm{A}$ & $34(21 \%)$ & 2 & 8 & $10(14 \%)$ & & \\
\hline \multirow[t]{2}{*}{ rFVIII-treated group } & Inh-negative (n=73) & \multicolumn{3}{|c|}{ Inh-positive ( $n=45)$} & & \\
\hline & & $\operatorname{LR}(n=16)$ & $H R(n=29)$ & $L R+H R(n=45)$ & OR & $95 \%$ Cl \\
\hline $\mathrm{G} / \mathrm{G}$ & $63(86 \%)$ & 15 & 23 & $38(84 \%)$ & 1.16 & $0.41-3.30$ \\
\hline $\mathrm{G} / \mathrm{A}+\mathrm{A} / \mathrm{A}$ & $10(14 \%)$ & 1 & 6 & $7(16 \%)$ & & \\
\hline \multirow[t]{2}{*}{ pdFVIII-treated group } & Inh-negative (n=90) & \multicolumn{3}{|c|}{ Inh-positive (n=27) } & & \\
\hline & & $\operatorname{LR}(n=8)$ & $H R(n=19)$ & $L R+H R(n=27)$ & OR & $95 \%$ CI \\
\hline $\mathrm{G} / \mathrm{G}$ & $66(73 \%)$ & 7 & 17 & $24(89 \%)$ & 0.35 & $0.09-1.24$ \\
\hline $\mathrm{G} / \mathrm{A}+\mathrm{A} / \mathrm{A}$ & $24(27 \%)$ & 1 & 2 & $3(11 \%)$ & & \\
\hline
\end{tabular}

fFVIII: recombinant factor VIII; pd FVIII: plasma-derived factor VIII; Inh: inhibitor; LR: low responder; HR: high responder; OR: odds ratio; CI: confidence interval 
T>C, p.Tyr795Tyr (rs1063857); c.2555 G>A, p.Arg852Gln (rs216321) and c.2880 G>A, p.Arg960Arg (rs1800380). The association between VWF:FVIIIB and SNP genotypes was assessed. The two silent SNP (p.Tyr795Tyr and p.Arg960Arg) (data not shown) and p.Thr789Ala had no impact on VWF:FVIIIB (Figure 1C). However, the c. $2555 \mathrm{G}>\mathrm{A}$ SNP, corresponding to the substitution of an arginine with a glutamine at position 852 , was associated with a statistically significant reduction in VWF:FVIIIB in the case of plasma from the heterozygous G/A patients as compared to plasma from patients with the frequent, homozygous $\mathrm{G} / \mathrm{G}$ genotype $(P<0.001,95 \%$ CI: 11.87-42.51) (Figure 1D). No patient with the rare A/A genotype was detected. Two patients carried either one of the p.Arg854Gln and p.Arg924Gln mutations associated with VWD with a heterozygous status. The transition p.Arg854GIn, described as a type $2 \mathrm{~N}$ VWD causative mutation, ${ }^{11}$ was found in one patient without inhibitors. Previously reported to be a polymorphism in a study of type $2 \mathrm{~N}$ VWD mutations, ${ }^{12}$ the p.Arg924Gln, which represents a non-conservative amino acid substitution in exon 21, was observed in one patient with inhibitors. These missense mutations were associated with normal VWF:Ag levels and reduced VWF:FVIIIB, $41 \%$ and $42 \%$ in one inhibitor-negative patient and one inhibitor-positive patient, respectively (lower 2 points in Figure 1A). A previous study by Nesbitt et al. identified the c. $2555 \mathrm{G}>\mathrm{A}$ SNP in 16 of 148 screened alleles. ${ }^{13}$ In
A

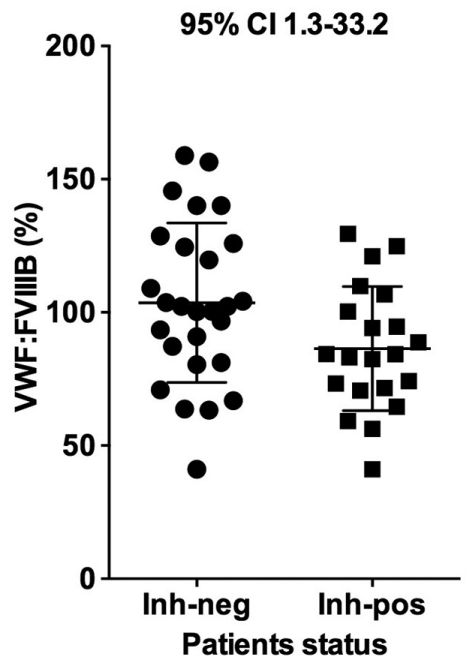

C

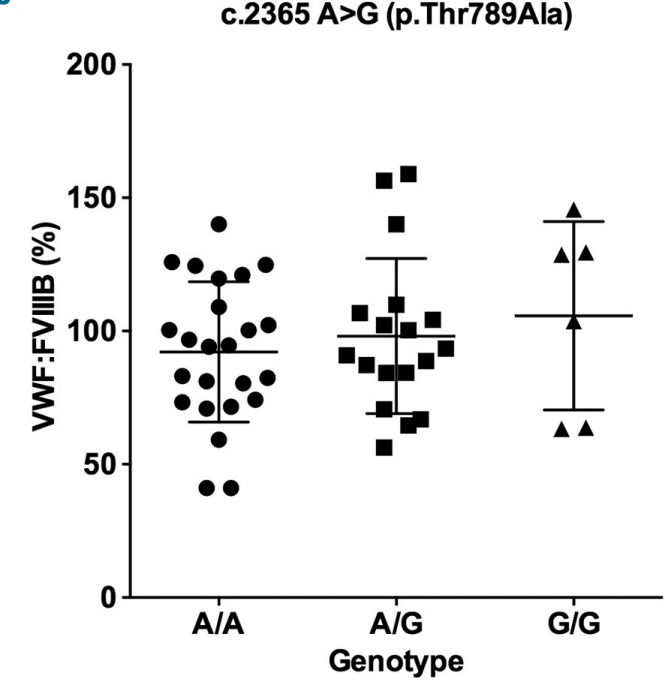

B

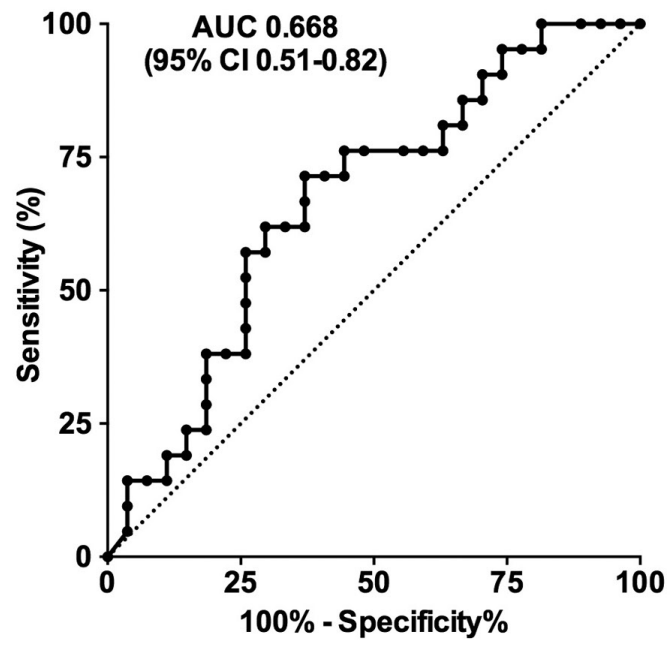

D

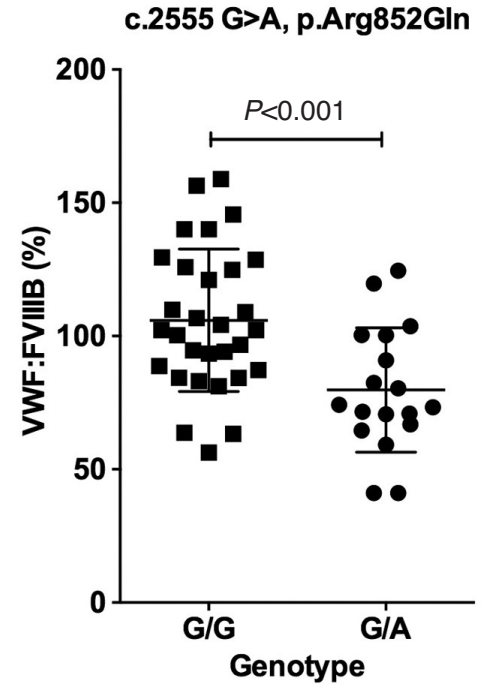

Figure 1. Relative endogenous von Willebrand factor binding and inhibitory status in patients with severe hemophilia A. (A) Association between relative von Willebrand factor binding (VWF:FVIIIB) and inhibitor status in patients with severe hemophilia A $(n=48)$. The $x$ axis represents the inhibitor status: patients with hemophilia A without FVIII inhibitor (Inh-neg) and with FVIII inhibitor (Inh-pos). The y axis represents the relative binding of recombinant FVIII to the endogenous VWF in the plasma of patients with severe hemophilia A measured by enzyme-linked immunosorbent assay (expressed in \%). The 95\% confidence intervals (95\% $\mathrm{Cl}$ ) was constructed with the standard errors derived from the Student $t$ distribution. (B) Receiver operating characteristic curve for predicting inhibitor development in patients with severe hemophilia A by measurement of VWF:FVIIIB. The true positive rate (sensitivity) is plotted as a function of the false positive rate (100-specificity). AUC: area under the curve. (C, D). Associations between VWF:FVIIIB and the p.Thr789Ala (c.2365 A>G) polymorphism (C) or the p.Arg852GIn (c.2555 G>A) polymorphism (D) in exon 18 of the VWF gene. Statistical differences were determined using the Student $t$-test. 
contrast to our findings, their results had suggested that VWF:FVIIIB was not affected by the p.Arg852Gln polymorphism in VWF, possibly because of the relatively low number of patients with the c. $2555 \mathrm{G}>\mathrm{A}$ SNP included in their study.

In an attempt to determine whether the c.2555 G>A SNP in exon 18 of the VWF gene is associated with the occurrence of FVIII inhibitors in patients, we searched for the SNP in 235 subjects enrolled in the SIPPET study. ${ }^{2}$ The cohort included 163 inhibitor-negative patients and 72 inhibitor-positive patients, encompassing 14 lowresponder and 48 high-responder patients. Genotype frequencies of the polymorphism are summarized in Table 1. The distribution of the c.2555 G>A genotypes did not deviate from the Hardy-Weinberg equilibrium in either inhibitor-negative or inhibitor-positive patients. No clear association between the c. $2555 \mathrm{G}>\mathrm{A}$ SNP genotypes and the development of inhibitors was observed (odds ratio, 0.61; 95\% CI: 0.28-1.32) (Table 1). These data are in line with those derived from a similar analysis performed in parallel using biological samples from a multicenter retrospective cohort of 281 patients with severe hemophilia $\mathrm{A}^{14}$ (Online Spplementary Tables S1 and S2), suggesting that the different ethnic origin of patients in the SIPPET cohort $^{2}$ does not account for the results. Genotypes and allele frequencies in both cohorts were identical to those in the 1000 Genomes Project $(1111 \mathrm{G}) .^{8}$

If our working hypothesis is correct, the nature of the VWF variant should play a role predominantly in patients receiving recombinant FVIII products, but not in patients receiving exogenous VWF with plasma-derived FVIII products. Among the 235 SIPPET patients included in the present study, 118 patients were treated with recombinant FVIII concentrates and 117 patients received plasma-derived FVIII products following randomization (1:1). Associations between the genotype distribution and development of FVIII inhibitors were investigated in the two groups of patients (Table 1). There was again no clear association between the presence of the A allele of the c. $2555 \mathrm{G}>\mathrm{A}$ SNP and the presence of a FVIII inhibitor, either in the case of the group treated with recombinant FVIII (OR, $1.16 ; 95 \%$ CI: $0.41-3.30)$ or in the case of the group treated with a plasma-derived FVIII product (OR, 0.12 ; $95 \%$ CI $0.09-1.24)$. A genome-wide association study evaluated 13,331 SNP from 1,081 genes using the Illumina iSelect platform for associations with inhibitor development in patients with hemophilia A. The study group included 833 subjects from three independent cohorts. The authors identified 53 SNP as significant predictors of inhibitor status, thus highlighting the complexity of the anti-FVIII immune response. ${ }^{15}$ However, the genome-wide association study did not find associations of SNP in the VWF gene with the inhibitor status of the patients, which supports the present findings.

A major limitation of this study is the discrepancy between our observations: (i) an overall reduced relative endogenous VWF binding in the plasma from inhibitorpositive patients with severe hemophilia A; (ii) a reduced relative endogenous VWF binding with the c.2555 G>A SNP; and (iii) the lack of association of the 2555 G $>$ A SNP with the inhibitory status of the patients. Recently, Muczynski et al. developed a recombinant FVIII (FVIII$\mathrm{KB} 013 \mathrm{bv}$ ) that contains two VWF-specific nanobodies in place of the B domain. ${ }^{16}$ FVIII-KB013bv has a 25 -fold increased affinity for VWF as compared to B domaindeleted FVIII, and exhibited a prolonged residence time in the blood of FVIII-deficient mice. Interestingly, FVIII$\mathrm{KB} 013 \mathrm{bv}$ demonstrated an almost complete lack of immunogenicity in vivo in FVIII-deficient mice. In view of the latter information, the discrepancy between our observations may be explained by the fact that, owing to the multi-causal nature of the inhibitor risk, an affinity of the endogenous VWF for therapeutic FVIII in the high physiological range does not systematically play a major protective role. Instead, stabilization of the complex beyond the physiological equilibrium affinity is required to exert blatant immune-protective functions.

Yohann Repessé, ${ }^{1,2,3}$ Catherine Costa, ${ }^{4 \dagger}$ Roberta Palla, ${ }^{5}$ Elika Farrokhi Moshai, ${ }^{6}$ Annie Borel-Derlon, ${ }^{1,2,3}$

Roseline D'Oiron, ${ }^{7}$ Chantal Rothschild, ${ }^{8}$ Amal El-Beshlawy, Mohsen Elalfy, ${ }^{10}$ Vijay Ramanan, ${ }^{11}$ Peyman Eshghi, ${ }^{12}$

Johannes Oldenburg, ${ }^{13}$ Anna Pavlova, ${ }^{13}$ Frits $R$ Rosendaal, ${ }^{14}$ Flora Peyvandi, Srinivas V. Kaveri and Sébastien LacroixDesmazes ${ }^{6}$

${ }^{+}$This work is dedicated to the memory of our late colleague and friend Catherine Costa, an extraordinary geneticist and a wonderful human being.

'CHU de Caen, Hematology Laboratory, Caen, France; INSERM, U1237, GIP Cyceron, Caen, France; ${ }^{3}$ Normandie Université, UNICAEN, UFR Santé, Caen, France; ${ }^{4}$ Service de Génétique et Biologie Moléculaires, APHP, Groupe Hospitalier Cochin Broca, Hôtel-Dieu, Site Cochin, Paris, France; ${ }^{5}$ Department of Pathophysiology and Transplantation, Università degli Studi di Milano, Milano, Italy; ${ }^{6}$ Centre de Recherche des Cordeliers, INSERM, Sorbonne Université, USPC, Université Paris Descartes, Université Paris Diderot, Paris, France; ${ }^{7}$ Centres de Traitement de l'Hémophilie, APHP, Le KremlinBicêtre, France; ${ }^{8}$ Centres de Traitement de l'Hémophilie, APHP, Hôpital Necker, Paris, France; ${ }^{9}$ Pediatric Hematology Department, Cairo University Pediatric Hospital, Cairo, Egypt; ${ }^{10}$ Faculty of Medicine, Ain Shams Center, University - Department of Pediatrics, Cairo, Egypt; "1ehangir Clinical Development Center, Department of Hematology, Jehangir Hospital Premises, Pune, India; ${ }^{12}$ Pediatric Congenital Hematologic Disorders Research Center, Shahid Beheshti University of Medical Sciences, Tehran, Iran; ${ }^{13}$ Institute of Experimental Hematology and Transfusion Medicine, University Clinic Bonn, Bonn, Germany and ${ }^{14}$ Department of Clinical Epidemiology, Leiden University Medical Center, Leiden, the Netherlands.

Funding: this work was supported by Institut National de la Santé et de la Recherche Médicale (INSERM), Centre National de la Recherche Scientifique (CNRS), Université Paris Sorbonne, grants from CoMETH-Recherche 2016, and Agence Nationale de la Recherche (ANR-07-MRAR-028-01).

Appendix: SIPPET Study Group. S. Hanagavadi, Davangere, India; R. Varadarajan, Chennai, India; M. Karimi, Shiraz, Iran; M. V. Manglani, Mumbai, India; C. Ross, Bangalore, India; G. Young, Los Angeles, USA; T. Seth, New Delhi, India; S. Apte, Pune, India; D. M. Nayak, Karnataka, India; E. Santagostino, M. Elisa Mancuso, Milan, Italy; A. C. Sandoval Gonzalez, Monterrey, Mexico; J. N. Mahlangu, Johannesburg, South Africa; S. Bonanad Boix, Valencia, Spain; M. Cerqueira, Rio de Janeiro, Brazil; N. P. Ewing, Duarte, USA; C. Male, Vienna, Austria; T. Owaidah, Rivadh, Saudi Arabia; V. Soto Arellano, Fargo, USA; N. L. Kobrinsky, Jackson, USA; S. Majumdar, and

R. Perez Garrido, Sevilla, Spain; A. Sachdeva, New Delhi, India; M. Simpson, Chicago, USA; M. Thomas, Kerala, India; E. Zanon, Padova, Italy; B. Antmen, Adana, Turkey; K. Kavakl, Izmir, Turkey; M. J. Manco-Johnson, Aurora, USA; M. Martinez, Buenos Aires, Argentina;

E. Marzouka, Santiago, Chile; M. G. Mazzucconi, Rome, Italy; D. Neme, Buenos Aires, Argentina; A. Palomo Bravo, Malaga, Spain; R. Paredes Aguilera, Mexico City, Mexico; A. Prezotti, Vitoria, Brazil; K. Schmitt, Linz, Austria; B. M. Wicklund, Kansas City, USA; B. Zulfikar, Istanbul, Turkey.

Correspondence: SEBASTIEN LACROIX-DESMAZES.

Sebastien.Lacroix-Desmazes@crc.jussieu.fr

doi:10.3324/haematol.2018.212001 
Information on authorship, contributions, and financial \& other disclosures was provided by the authors and is available with the online version of this article at www. haematologica.org.

\section{References}

1. Lai J, Hough C, Tarrant J, Lillicrap D. Biological considerations of plasma-derived and recombinant factor VIII immunogenicity. Blood. 2017;129(24):3147-3154.

2. Peyvandi F, Mannucci PM, Garagiola I, et al. A randomized trial of factor VIII and neutralizing antibodies in hemophilia A. N Engl J Med. 2016; 374(21):2054-2064

3. Calvez T, Chambost H, d'Oiron R, et al. Analyses of the FranceCoag cohort support differences in immunogenicity among one plasmaderived and two recombinant factor VIII brands in boys with severe hemophilia A. Haematologica. 2018;103(1):179-189.

4. Gangadharan B, Ing M, Delignat S, et al. The C1 and C2 domains of blood coagulation factor VIII mediate its endocytosis by dendritic cells. Haematologica. 2017;102(2):271-281.

5. Meeks SL, Cox CL, Healey JF, et al. A major determinant of the immunogenicity of factor VIII in a murine model is independent of its procoagulant function. Blood. 2012;120(12):2512-2520.

6. Leyte A, Verbeet MP, Brodniewicz-Proba T, van Mourik JA, Mertens $\mathrm{K}$. The interaction between human blood-coagulation factor VIII and von Willebrand factor. Biochem J. 1989;257:679-683.

7. Bittar LF, de Paula EV, Mello TB, et al. Polymorphisms and mutations in vWF and ADAMTS13 genes and their correlation with plasma levels of FVIII and vWF in patients with deep venous thrombosis. Clin Appl Thromb Hemost. 2011;17(5):514-518.

8. Wang OY, Song J, Gibbs RA, et al. Characterizing polymorphisms and allelic diversity of von Willebrand factor gene in the 1000 Genomes. J Thromb Haemost. 2013;11(2):261-269.

9. Gouw SC, van den Berg HM. The multifactorial etiology of inhibitor development in hemophilia: genetics and environment. Semin Thromb Hemost. 2009;35(8):723-734.

10. Eckhardt CL, van Velzen AS, Fijnvandraat CJ, van der Bom JG. Dissecting intensive treatment as risk factor for inhibitor development in haemophilia. Haemophilia. 2016;22(3):e241-244.

11. Gaucher C, Mercier B, Jorieux S, Oufkir D, Mazurier C. Identification of two point mutations in the von Willebrand factor gene of three families with the 'Normandy' variant of von Willebrand disease. Br J Haematol. 1991;78(4):506-514.

12. Hilbert L, Jorieux S, Proulle V, et al. Two novel mutations, $\mathrm{Q} 1053 \mathrm{H}$ and C1060R, located in the D3 domain of von Willebrand factor, are responsible for decreased FVIII-binding capacity. Br J Haematol. 2003;120(4):627-632.

13. Nesbitt IM, Goodeve AC, Preston FE, Peake IR. von Willebrand factor/factor VIII binding is not affected by the Arg89Gln polymorphism in von Willebrand factor. Thromb Haemost. 1996;76(5):820821.

14. Repesse Y, Peyron I, Dimitrov JD, et al. Development of inhibitory antibodies to therapeutic factor VIII in severe hemophilia A is associated with microsatellite polymorphisms in the HMOX1 promoter. Haematologica. 2013;98(10):1650-1655.

15. Astermark J, Donfield SM, Gomperts ED, et al. The polygenic nature of inhibitors in hemophilia A: results from the Hemophilia Inhibitor Genetics Study (HIGS) Combined Cohort. Blood. 2013;121(8):14461454

16. Muczynski V, Casari C, Moreau F, et al. A factor VIII-nanobody fusion protein forming an ultrastable complex with VWF: effect on clearance and antibody formation. Blood. 2018;132(11):1193-1197. 